\title{
Cancer screening: concerns, controversy and evidence
}

\section{Sarah McGilla,c and Stacy M Carter ${ }^{b, c, d}$}

a Screening and Prevention, Cancer Institute NSW, Sydney, Australia

${ }^{\mathrm{b}}$ Centre for Values, Ethics and the Law in Medicine, University of Sydney, NSW, Australia

' Guest Editor, Issue 3, 2017

d Corresponding author: stacy.carter@sydney.edu.au

\section{Article history}

Publication date: July 2017

Citation: McGill S, Carter SM. Cancer screening: concerns, controversy and evidence. Public Health Res Pract. 2017;27(3):e2731720. https://doi.org/10.17061/phrp2731720
Cancer accounts for approximately one-fifth of the total disease burden in Australia, with $94 \%$ of this burden due to premature death. ${ }^{1}$

Depending on the cancer type, and the quality and appropriateness of screening protocols, screening programs can prevent such premature death, reduce numbers of people with advanced disease, enable more conservative clinical management and improve survivorship outcomes. However, some forms of screening can also cause significant harms, which need to be weighed against the expected benefits.

This issue of Public Health Research \& Practice includes articles that review the evidence for, and history of, organised cancer screening programs in Australia, and consider other cancers for which less formalised but still frequent testing occurs. The evidence for benefits from organised screening programs is considered, along with the evidence for harms in asymptomatic, well populations.

Australia has three population screening programs: the National Cervical Screening Program, BreastScreen Australia and the National Bowel Cancer Screening Program. Olver and Roder discuss these three programs and consistent with other discussion in this issue - find that the focus of the three screening programs should be to inform screening participants of the evidence, maximise reductions in mortality and minimise adverse effects. This article provides a useful background and notes the possibility of genomic testing in future population-based cancer screening in Australia.

An evidence based approach to cancer screening is essential. However, assessing the efficacy and effectiveness of screening programs is a complex and often contentious process, as described by Jacklyn et al. Understanding the evidence, and weighing the positive and negative effects given the range of views can be challenging for both health professionals and consumers; this article provides guidance on common pitfalls.

In an 'In discussion' paper, leading specialists examine one of the most contested issues in cancer screening - prostate-specific antigen (PSA) testing of men at normal risk of prostate cancer. Despite recent new trials and policy development, there is still considerable contention about this practice. A commonly proposed solution is to ensure that men are well informed before they decide whether to have a PSA test. On the face of it, this seems reasonable, but it may not be easily achieved: careful evaluation of the evidence 
and sophisticated communication will be needed for men to gain a balanced understanding to support their decision making.

Hersch et al. consider what might be required to achieve such informed decision making about screening, discussing strategies such as decision aids and shared decision making. Providing balanced and instructive information in an accessible format across the diversity of consumers is complicated. An important start is seeking to understand what matters to individuals, including their perceptions of risk and what counts as quality of life.

A potential harm in some forms of cancer screening is overdiagnosis. As Carter and Barratt describe, when overdiagnosis occurs, it can harm healthy people, may create opportunity costs by misdirecting healthcare resources, and potentially undermines the ultimate goal of screening, which should be to reduce suffering and premature death. In weighing up the outcomes of screening, it is important to consider all the harms averted by screening (e.g. all the premature deaths likely to be prevented) and also all the harms caused by screening (e.g. all the people likely to be overdiagnosed and treated, or receiving a false-positive result).

Decision making about screening is challenging for consumers and policy makers, perhaps more now than ever before. This special issue aims to progress the conversation and to provide useful resources that the public health community can use in tackling this ongoing challenge.

We hope readers enjoy these themed papers and all the articles in this issue. Public Health Research \& Practice aims to deliver engaging, high-quality, peer-reviewed articles from Australian and international thought leaders, policy makers, practitioners and researchers that will inspire, provoke debate and be useful in your work.

\section{Competing interests}

$\mathrm{SM}$ is employed as Director of Screening and Prevention at the Cancer Institute NSW and is program manager for the three national screening programs in Australia. SC received travel funding related to her work on cancer overdiagnosis from the Preventing Overdiagnosis Conference 2017, hosted by the Quebec Medical Association.

\section{Author contributions}

SM and SC both contributed to writing and editing the article.

\section{References}

1. Australian Institute of Health and Welfare. Burden of cancer in Australia: Australian burden of disease study 2011. Canberra: AlHW; 2017 [cited 2017 Jul 12]. Available from: www.aihw.gov.au/WorkArea/DownloadAsset. aspx?id=60129559772

\section{Copyright: (c) (7)(2) (2)}

(C) 2017 McGill and Carter. This article is licensed under the Creative Commons Attribution-NonCommercial-ShareAlike 4.0 International Licence, which allows others to redistribute, adapt and share this work non-commercially provided they attribute the work and any adapted version of it is distributed under the same Creative Commons licence terms. See: www.creativecommons.org/licenses/by-nc-sa/4.0/ 